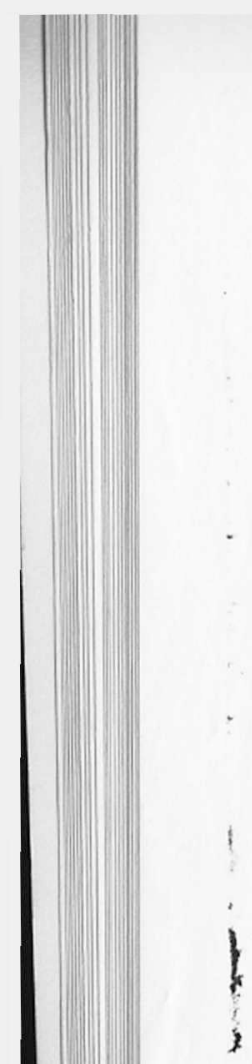

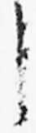

; 


\section{DER SCHWERTGOTT IN YAZILIKAYA}

Kurt Bittel, Heidenheim.

Vor mehr als zwanzig Jahren schrieb Ekrem Akurgal in seinem Buch über die Kunst der Hethiter: "Noch unklar ist die Bedeutung des Schwertgottes, der wegen der Vielzahl der Hörner und der Gottesideogramme, die seine Spitzmütze schmücken, als eine wichtige Gottheit angesehen werden muss. Das Relief ist zwar nichts anderes als die Umsetzung eines Kleinkunstmotives in die monumentale Dimension, doch in der Vorstellung des Schwertgottes muss ein tiefer religiöser Sinn gesucht werden" (1). Trotz nicht geringer Bemühungen und Versuche, eine unanfechtbare Erklärung der ursprünglichen Bedeutung dieses merkwürdigen Reliefs (Yazılıkaya Nr. 82) in dem nahe der hethitischen Hauptstadt gelegenen Felsheiligtum zu erzielen, besteht der eben zitierte Satz Akurgals auch heute noch im wesentlichen zurecht. Die einleuchtendste Deutung, nämlich als "Schwert des Nergal" in Verbindung mit einem Totentempel ist streng genommen auch nur eine plausible Vermutung (2). Der eigentliche Beweis fehlt. Das Verständnis wird dadurch erschwert, dass das Relief in der hethitischen monumentalen Kunst ohne Beispiel ist und dass ihm eine Beischrift in Bilderschriftzeichen fehlt, die es präzis zu benennen erlaubten (Fig. 1). Darin steht das Relief, wenn man die ganze Serie von Yazllikaya in Betracht zieht, nicht allein. Die Zwölfgötter in Kammer A und in Kammer B (Nr. 1-12, 69-80), die den Himmel tragenden Stiermenschen (Kammer A, Nr. 28-29) und die beiden Löwenmenschen am Zugang zu Kammer B (Nr. 6768) sind ebenso schriftlos wie der Schwertgott (3). Es ist wohl erlaubt, dieses Fehlen so zu verstehen, dass es sich in allen Fällen, also auch bei Yazllikaya Nr. 82, um feststehende, im 13. Jahrhundert v. Chr. jedem Eingeweihten verständliche Inhalte handelte, die keiner schriftlichen Erklärung bedurften (4).

Während in der grossen Kammer (A) des Felsheiligtums alle Gottheiten, selbst das scheinbar isolierte Königsbild Nr. 64 (5), in gereihter Ordnung dargestellt sind, befinden sich die der kleinen Kammer (B) wie "Marken"' an den Felswändenangebracht, sind, wie immer wieder betont worden ist (6), scheinbar je für sich isoliert. Dies gilt für den äusseren Eindruck des mit den Zusammenhängen nicht vertrauten Betrachters. Dass aber auch sie doch einer einheitlichen Sinngebung unterworfen sind, ergibt 
sich sowohl aus ihrer Zugehörigkeit zu Unterwelt und Totenkult, wenn unsere vor Jahren geäusserte Deutung zutreffend ist, und dadurch, dass mindestens zwei von ihnen, die Zwölfgötter und der den König Tuthalija IV.führende Gott Sarrummazwar Bildvorstellungen sind,die nicht an die monumentale Kunst allein gebunden sind, sondern auch in der Kleinkunst vorkommen, in ihrer Ausrichtung einheitlich auf das nördliche Ende der Kammer ebenso wie der Schwertgott bezogen sind (7). Daraus wird trotz der räumlichen Isolierung untereinander die innere Bezogenheit deutlich, wenn ihr auch die bildliche fehlt, wie sie die Reliefs der Kammer A aufweisen.

Es mag erlaubt sein, noch kurz bei der inneren Ordnung der Kammer B zu verweilen. Rudolf Naumann hat 1938 vor der Nordwand dieser Kammer einen Felsblock bemerkt, der an der Rückseite bearbeitet ist, "weshalb er vielleicht als isolierte Basis eines Kultbildes gedeutet werden kann". Ausserdem fand sich wenig davon entfernt ein Werkblock mit einer Einarbeitung, den Naumann zu einer Basis zu ergänzen versucht hat (8). Es wurde dann gefolgert, dass diese Basis einem Kultbild gedient habe, das den eigentlichen Bezugspunkt der Kammer B bildete und für das die an der benachbarten Felswand angebrachte Kartusche Tuthalija's IV. bestimmt gewesen sei. Diese Vermutung erfuhr dadurch eine gewisse Stütze, dass im $3,5 \mathrm{~km}$ nordwestlich entfernten Dorfe Yekbas eine sekundär verwendete Standplatte gefunden worden ist, auf deren Oberseite, anscheinend als letzter Rest einer sehr grossen Statue, zwei menschliche Füsse erhalten sind. Peter Neve denkt sich diese Statue als eine komposite Grossplastik, hält es für nicht ausgeschlossen, dass es sich um ein hethitisches Werk handelt, und erwägt unter Berücksichtigung der Massverhältnisse der Platte und der eben erwähnten Basis in Yazılıkaya die Möglichkeit der Verschleppung aus der Kammer B des Felsheiligtums (9), umso mehr als von dort auch ein anderer, sicherer Fall von Dislozierung eines Monumentes in die unmittelbare Nähe des genannten Dorfes bekannt ist (10). Trăfen diese, wohlverstanden nur als Vermutung geäusserten, Annahmen das Richtige, wäre diese Platte mit den beiden Füssen der letzte erhaltene Rest jener postulierten Statue am Nordende der Felskammer, die den dominierenden Bezugspunkt, ja das eigentliche zentrale Wahrzeichen dieses Kammer gebildet haben soll. Mehr noch, man hätte in ihr, wenn man gelegentlich geäusserten Kombinationen mit der benachbart am Felsen angebrachten Königskartusche folgen will, ein Bildwerk zu sehen, das Tuthalija IV. darstellte. So schlüssig sich das ausnimmt, können doch wesentliche Bedenken nicht unerwähnt bleiben. 
Das Steinmaterial, aus dem diese Statue bestanden haben soll, ein violettbrauner, blasiger Basalt, ist zwar als natürliches Vorkommen in der Gegend von Boğazköy bezeugt, wäre aber für plastische, stationäre Werke ganz ungewöhnlich (11).Für solche Zwecke herrschte der Kalkstein weitaus vor, neben dem in wenigen Fällen Granit verwendet worden ist: Löwe vom Nişantaş und Löwen der Vorhalle des Adytons vom Tempel III in der Oberstadt (12). Die pantoffelartigen Schuhe, die der (oder die) Dargestellte trägt, überraschen etwas, weil man viel eher Schnabelschuhe erwarten würde, wie sie alle Götter und Könige in Yazılıkaya tragen (13), wenn sie nicht wie die beiden Löwenmenschen Nr. 67 und 68, von den Stiermenschen Nr. 28 und 29 ganz abgesehen, unbeschuht sind. Ohne Fussbekleidung ist allerdings auch das Relief eines Gottes am sog. Königstor in Hattuša, aber bei ihm sind die nackten Füsse durch die sorgfältige Wiedergabe der Zehen gekennzeichnet.

Das alles spricht nicht entscheidend gegen die vom Finder erwogenen Deutung, die ja, was noch einmal zu betonen ist, sowieso mit aller nötigen Reserve vorgetragen worden ist. Gravierender ist die von ihm und anderen, freilich ebenfalls nur als reine Vermutung ausgesprochene Identifizierung der postulierten Statue mit Tuthalija IV. für die der Name dieses Königs an der benachbarten Felswand zu sprechen scheint. Der Nachdruck muss dabei auf "Benachbart" liegen, denn diese Kartusche ist von der "Basis" am Nordende der Kammer B 2,8 m entfernt, zu viel, wie ich meine, als dass die Zusammengehörigkeit beider ohne Bedenken angenommen werden kann. Wäre nicht viel wahrscheinlicher der Königsname auf der Vorderseite der Basis der Herrscherstatue selbst zu erwarten und nicht räumlich erheblich davon getrennt an der Wand des Felsens, der mit dem Eingang zur Kammer C verbunden ist? Auf diesen Zugang ist sie bezogen, der, wie die Orientierung des Berggottes in dieser Kartusche nach Süden im Gegensinne aller anderen Darstellungen zeigt (14), der eigentliche Bezugpunkt dieser Kammer B gewesen ist und nicht eine hypothetische Königstatue. Der Name Tuthalija's IV. galt nach meiner Überzeugung dem Dromos zur Kammer C, mit anderen Worten dem, was die Bedeutung dieser natür!ichen Felsnische, was ihren Inhalt, besser ausgedrückt: ihren Gehalt, ausgemacht hat.

So problematisch nach wie vor die Deutung dieses Reliefs des "Schwertgottes", das auf keinen besinnlichen Besucher in unseren Tagen seinen Eindruck verfehlt, noch ist und so wenig Hilfe der Archäologe bei den an die verfügbaren Texte gebundenen Hethitologen auch vorläufig findet, so hatte Ekrem Akurgal doch völlig recht mit seiner Auffassung, dass man es mit der "Umsetzung eines Kleinkunstwerkes in die monumen- 
tale Dimension" zu tun habe. Das kann sich freilich zunächst nur auf die formale Seite, nicht aber zugleich auf Bedeutung und Inhalt beziehen. Eben zur Form aber lässt sich heute dank einiger Neufunde etwas mehr sagen. Über die Gestalt von hethitischen Langschwertern, denn nur ein solches kann mit Yazllıkaya 82 gemeint sein, ist man freilich nach wie vor schlecht unterrichtet. Kein originales Exemplar ist bisher aufgefunden worden. Doch hatte diese Schwertform zweifellos in Anatolien eine alte Tradition, was Exemplare aus einer Spätphase der Frühen Bronzezeit vom Arslantepe bei Malatya und auch das längst bekannte Schwert aus dem Grab M.A. in Alaca-Höyük beweisen. Alle mit einer Waffe dargestellten Götter und Könige in Yazılıkaya, tragen, soweit sie nicht Sichelschwerter führen, am Ende leicht aufgebogene Kurzschwerter mit mondsichelförmigem Griffende (15). Die Bildwerke aus der Zeit des sog. hethitischen Grossreiches von anderen Orten, soweit Bewaffnete dargestellt sind, zeigen die gleiche Waffe. Werke der Steinschneidekunst verhalten sich darin nicht anders. Aber es gibt Ausnahmen. Die beiden männlichen Gestalten der Felsreliefs in Gâvurkale haben Schwerter mit zwar gleicher Griffkonstruktion, jedoch mit einer Klinge, die sie von den übrigen erwähnten infolge der gestreckten Form abhebt. Das gilt vielleicht auch für das Relief von Hemite, sicher für den Wettergott der Stele von Akçaköy, bei der die Schwertscheide in ein dreigeteiltes Ende ausläuft, eine Art Fransen, im Angleich an den unteren Saum des Leibrockes. In keinem Fall, weder in Gâvurkale, noch in Akçaköy, erreichen aber diese Schwerter die Grösse wirklicher Langschwerter, noch die Proportionen des Schwertes in Yazıl1kaya. Keines, weder die kürzere, noch die längere Form, zeigt ganz oder zu Teilen auch nur die geringste figurale, plastische Ausgestaltung. Das Emblem im Felsheiligtum steht darin bisher völlig allein. Aber man kennt neuerdings wenigstens Verwandte.

Eine allseitig mit Vogelköpfen, Löwengreifen, Löwenprotomen und Flügellöwen, einer allegorischen Darstellung des Sonnengottes förmlich überzogene, dem Vernehmen nach bei Şarkışla gefundene Ceremonialaxt zeigt deutlich genug, wie weit solche an sich in ihrer Grundform einfache Waffen oder Geräte in den Bereich von Religion und Kult gehörenden Darstellungen dienen konnten,wenn ihre Bestimmung nicht profaner Art gewesen ist(16). Vom gleichen Ort (17) und aus dem gleichen Gesamtfund stammt auch eine bronzene Lanzenspitze von insgesamt $33,9 \mathrm{~cm}$ Länge mit sehr dünnem, an der Grösse des Ganzen gemessen fast hauchdünnem Blatt (Fig. 2 a-b).Ohne Zweifel ist es eine Lanze nicht für den Kampf, sondern für die Jagd, und zwar eine für die Jagd auf Schwarzwild bestimmte Saufeder wie der Fachausdruck lautet. Dem entspricht die figurale 
Ausgestaltung, die aus zwei massiv gegossenen Eberprotomen beidseitig eines Tüllenrings besteht, der über das Schäftungsende des Blattes geschoben und dort vernietet ist. In allen Einzelheiten entsprechen sich die Protome vollkommen: dem scharfen Halskamm, den abstehenden erhobenen Ohren, dem Rüssel, den Hauern und dem auf den Vorderbeinen liegenden Kopf. Im Bezug auf das Ganze nehmen sie ein den beiden Löwenprotomen des Schwertgottes sehr ähnliche Position ein. Zu dieser Gruppe gesellt sich auch die wohlbekannte Axt aus Ras Shamra mit einer Eberprotome am bronzenen Schaftloch und zwei symmetrisch zum eisernen Blatt ausgerichteten Löwenköpfen, die ebenfalls als Protome zu verstehen sind (18). Noch näher steht der Saufeder von Şarkışla eine Lanzenspitze aus Bronze, ebenfalls von Ras Shamra, die genau wie das anatolische Exemplar am Schaftende einen Ring mit zwei Eberköpfen besitzt. Das Blatt der Lanze ist aber nicht breit und dünn, sondern langgestreckt, schmal und dazu längs profiliert (19). Mit dieser Eigenschaft entspricht sie einem ebenfalls zum Fund von Şarkışla gehörigen, 47,5 cm langen, sehr schweren (1 Kilo 75 gr), massiv gegossenen Stück mit achteckiger, geschlitzter Tülle, die bis zur Spitze durchgeht, jedoch in der Partie des Blattes scharfkantig profiliert und von der eigentlichen Blattschneide durch eine besondere Profilstufe abgesetzt ist (Fig. 3 a-c). Das schmale, sehr scharfe Blatt wächst an der Basis in zwei Absätzen, also recht kunstvoll, aus der Tülle heraus. Die beiden zuletzt erwähnten Lanzenspitzen von Ras Shamra und Şarkışla sind deshalb in diesem Zusammenhang von Belang, weil sie zeigen, dass es Waffen mit stark profiliertem Blatt gab, genau wie es auch die Klinge des Schwertgottes mit ihrem betonten Mittelgrat aufweist. Es versteht sich fast von selbst, dass damit eine Klinge aus Bronze gemeint ist, kaum aus Eisen wie einmal vermutet wurde (20).

Sieht man alle aufgeführten Objekte mit schon früher in diesem Zusammenhang berücksichtigten (21),ergibt sich daraus, dass die figurale Ausgestaltung von Waffen, Axten ur,d Lanzen, in dem Zeitraum, in dem auch Yazılıkaya seinen Ort hat, nicht ungewöhnlich war. Dabei ist es nicht erheblich, ob dieser Brauch mehr hurritischen, wie gelegentlichangenommen wird, oder hethitischen Ursprungs gewesen ist. Auf jeden Fall kulminierter im Schwertgott in Yazılıkaya in einem Werk, das von am hethitischen Hofe von Hattuša im 13. Jahrhundert lebendigen Vorstellungen getragen gewesen sein muss. Dabei ist noch ein weiteres Denkmal zu erwähnen.Der Schwertgott gliedert sich in Kammer B des Felsheiligtums in eine Schöpfung ein, die auf TuthalijaIV. zurückgeht, sei es dass er sie zu seinen Lebzeiten veranlasst hat, sei es dass sie von seinem unmittelbaren Nachfolger als Monument für den Vater ausgestattet worden ist. Eben von Tut- 
halija IV. kennt man eine ungewöhnlich grosse Zahl von Varianten der Ausgestaltung seiner Namenskartusche, darunter eine, erst kürzlich auf einer in der Oberstadt von Boğazköy entdeckten Stele, bei der der grosskönigliche Name links und rechts von Löwenprotomen flankiert ist (22), die gewiss ebensowenig wie die des Schwertgottes lediglich dekorativ zu verstehen sind, sondern die Kraft und Macht des Dynasten mit zum Ausdruck bringen sollen.

Die Bezeichnung "Schwertgott" für das Relief Nr. 82 in Yazllkaya, die sich seit mehr als einem halben Jahrhundert eingebürgert hat, ist deshalb berechtigt, weil der obere Griffabschluss in einem Kopf besteht, der durch seine hohe Mütze deutlich als Gott gekennzeichnet ist. Ob freilich zugleich als besonders hohen Ranges, wie man gemeint hat, ist von der Darstellung nicht ablesbar. Wenn nämlich die Zahl der Hörner an der Mütze für den Rang wirklich verbindlich sein sollte, was nicht eindeutig feststeht, kann eine solche Geltung in diesem Falle nicht in Anspruch genommen werden, weil entgegen meiner früheren und auch Ekrem Akurgal's Meinung (23) nicht mehrere, sondern lediglich ein einziges Horn am vorderen Mützenrand vorhanden ist. Vermutlich kam es hier auch gar nicht so sehr auf den allgemeinen Rang dieses Gottes im hethitischen Pantheon an, sondern auf seine Singularität, auf die Geltung und die ganz spezielle Rolle, die ihm nach der Auffassung der durch Tuthalija IV. und Suppiluliuma II. determinierten Generationen der hethitischen Dynastie des Grossreiches im Verbande der Kammer B zukamen. Ob damit wirklich eine mit Tod und Unterwelt, ähnlich dem babylonischen Nergal (24),verbundene Gottheit gemeint ist, oder ein auf die beiden erwähnten Könige individuell ausgerichtetes numen, werden hoffentlich uns heute noch versagte Quellen eines Tages lehren.

\section{ANMERKUNGEN (NOTES)}

1). Ekrem Akurgal, Die Kunst der Hethiter (München 1961) 87.

2). Das hethitische Felsheiligtum Yazlıkaya (Berlin 1975) 255 (im weiteren zitiert: Yaz.). K. Bittel, Hattuscha, Hauptstadt der Hethiter (Koln 1983) 158 f.

3). Auch der Berggott Nr. 13 ist anscheinend ohne Beischrift (Yaz. 128 und 179). E.Masson will dagegen hier die Legende dTá-sà gesehen haben (Le panthéon de Yazllikaya, nouvelles lectures, Paris 1981, 32 f.mit P1.XVI 13), was H.G.Güterbock bei einer Nachprüfung am Ort nicht bestattigen konnte (Les hieroglyphes de Yazlikaya, a propos Die Sache bedarf einer erneuten Prufung am Felsen. Ohne Beischriften sind auch die männlichen Götter Nr. 18-22 (Yaz. 178, H.G.Güterbock). E. Masson glaubt jedoch auch hier, d.h.bei 18,20,22- nich mehr bei 21 - Spuren von Zeichen wahrgenommen zu haben (a. O.27 f.mit P1. XIII), die H.G.Güterbock aber auf Grund neuer Autopsie nicht anerkennt (a.O.37 f.: "This reliefs have no inscriptions"). Seine Feststellung, dass an den entscheidenden Stellen die Felswand vollig glatt sei, kann man nur bestattigen. Andererseits ist es aber verständlich, dass E. Masson 1978 briefl. im Hinblick auf diese Reihe Das haben wohl auch andere empfunden. Ich überlegte früher gelegentlich, ob hier die ursprünglichen Namen einmal abgemeisselt und durch neue, aufgemalte ersetzt worden seien. Aber diese Möglichkei ist kaum ernsthaft in Erwägung zu ziehen. Eher kőnte man daran denken, dass diese Fünf (Nr. 18-22) mit den Gottheiten Nr. 23 und 24, die diesen unmittelbar vorangehen und offenbar keine Beischriften, sondern "ein Zeichen oder Symbol" ohne Gotteszeichen jeweils uber der Hand haben (Yaz. $178 \mathrm{mi}$ Taf. 17,2 und 56), eine Einheit bildeten und so gelüufig waren, dass sie keiner erklärenden Beischrift Entscheidung ist vorlaufig nicht moglich.

4). Vgl. dazu Yaz. 186 im Hinblick auf unbeschriftete Reliefs: “Vielleicht war auch die Position innerhalb der Reihe, Zwischen sicher identifizierten Figuren, schon allein ausreichen für die Benennung (H.G.Güterbock)

5). Yaz. 145 unten und 253.

6). Yaz. 160,255

7). Das gilt selbst fur das von mir vermutete unfertige, nur in grober Bosse angelegte Relief unmittelba nördlich vom Schwertgott an derselben Felswand (Yaz. 164), denn auch diese Figur wăre, wie der Umriss zeigt (Yaz. Taf. 52,2), nicht anders als Nr. 69-80,81 und 82 nach Norden zu orientiert gewesen. Albrecht Goetze hat ubrigens bei zweimaliger Erörteung an Ort und Stelle jeweils die Moglichkeit, das. hier ein weiteres Relief in den ersten Anfangen stecken geblieben sei, nicht gelten lassen. Ich halte jedoch trotzdem an dieser Deutung fest.

8). Alle Einzelheiten in Yaz. 43 f.mit Abb. 16-17. Zur Lage vgl. den Plan Abb. 26 auf S.50 ("Basis").

9). Archăologischer Anzeiger 1982,389 ff.mit Abb. 8-10 (P.Neve).

10). Yaz. 144 f., 181 Taf. 36, 2-3.

11). Bei kleineren Freiplastiken mag Basalt gelegentlich verwendet worden sein, zum Beispiel bei dem Vogelkopf WVDOG 60,14 mit Taf. 1,3, wenn diese Plastik wirklich von Boğazkőy stammt und hethitisch ist.

12). WVDOG 60, 11 mit Taf. 8, 2-4

13). Zusammengestellt Yaz. Taf. 64 unten

14). Yaz. Taf. $52,1,3$

15). Yaz. Taf. 65, 34-81. 
16). K.Bittel, Beitrag zur Kenntnis hethitischer Bildkunst (Sitzungsberichte der Heidelberger Akademie der Wissenschaften, Phil. -hist. Klasse 1976,4) $19 \mathrm{ff}$.mit Taf. VII-XII. Ebenda 26 ist auch auf Textstellen hingewiesen, die wenigstens teilweise solche figural verzierten Gerăte und Waffen bezeugen.

17). Zu seiner Lage und Bedeutung siehe Istanbuler Mitteilungen 25, 1975, $309 \mathrm{ff}$.

18). Claude F.-A. Schaeffer, Mission de Ras Shamra III, Ugartica 1, Paris 1939, 108 ff. mit P1. XXII.

19). Schaeffer a.0.113 Fig. 104 und PL. XXIII.

20). Paul Couissin, Le Dieu-Epée de Iasili-Kaïa et le culte de l'épée dans l'antiquité (Revue Archéologique 5 sér., XXVII 1928) 114. Dabei muss alerdings zugestanden werden, dass anderwărts, zum Beispiel in der mitteleuropaischen Hallstattkultur, die eisernen Langschwerter zunăchst auch in der Gestalt der Klinge den bronzenen angeglichen sind.

21). WVDOG 61, Leipzig 1941, 103. Antithetisch postierte Löwen kommen in Anatolien schon auf Siegeln der 1. Hälfte des 2. Jahrtausends vor, wie das Tizskiewitz- und das sog. Aydın Siegel mit den beiden aufgerichtet stehenden Tieren (gute Abbildung bei M.J. Mellink siehe Anm. 22) und auch Siegel vom Karahöyük bei Konya zeigen (Sedat Alp, Zylinder- und Stempelsiegel aus Karahőyük bei Konya, Ankara 1968, $193 \mathrm{Nr} .108$ mit Abb. 105). Auch die hăngenden Lowen am Griff eines Schwertes, das aus der Gegend von Diyarbakır stammt und der Zeit um 1800 zugewiesen wurde, ist, worauf schon Früher avfmerksam gemecht worden ist, in diesem Zusammenhang zu Erwăhnen.

22). Archäologischer Anzeiger 1984, 337 Abb. 10 (P.Neve). K.Bittel, Denkmäler eines hethitischen Grosskönigs des 13. Jahrhunderts vor Christus, Oplanden, 1984, 27 mit Taf. XVI.

23). WVDOG 61, 101 (K.Bittel); Ekrem Akurgal, Die Kunst der Hethiter, München 1961, 87.

24). Es ist bemerkenswert, dass schon Georges Perrot vor fast hundert Jahren, freilich aus etwas anderen Überlegungen, auch an Nergal dachte (Perrot-Chipiez, Histoire de l'Art dans l'Antiquité, Paris 1887, 697). ... Bei einem senkrecht im Kammerboden steckenden Schwert kommen dem Suchenden auch die drei parallel aufgerichteten, allerdings offenbar frei schwebenden Schwerter auf der vollstăndig von mythologischen Motiven bestimmten Goldschalr von Hasanlu als vage Affinităt in den Sinn (Edith Porada, Alt-Iran, die Kunst in vorislamischer Zeit, Baden-Baden 1962, 86 Fig. 60; M. J.Mellink, The Hasanlu Bowl in Anatolian Perspective, in: Iranica Antiqua V1 1966, 73 Fig. 1 b. Beidemal sind aber die Schwerter nicht behandelt). 


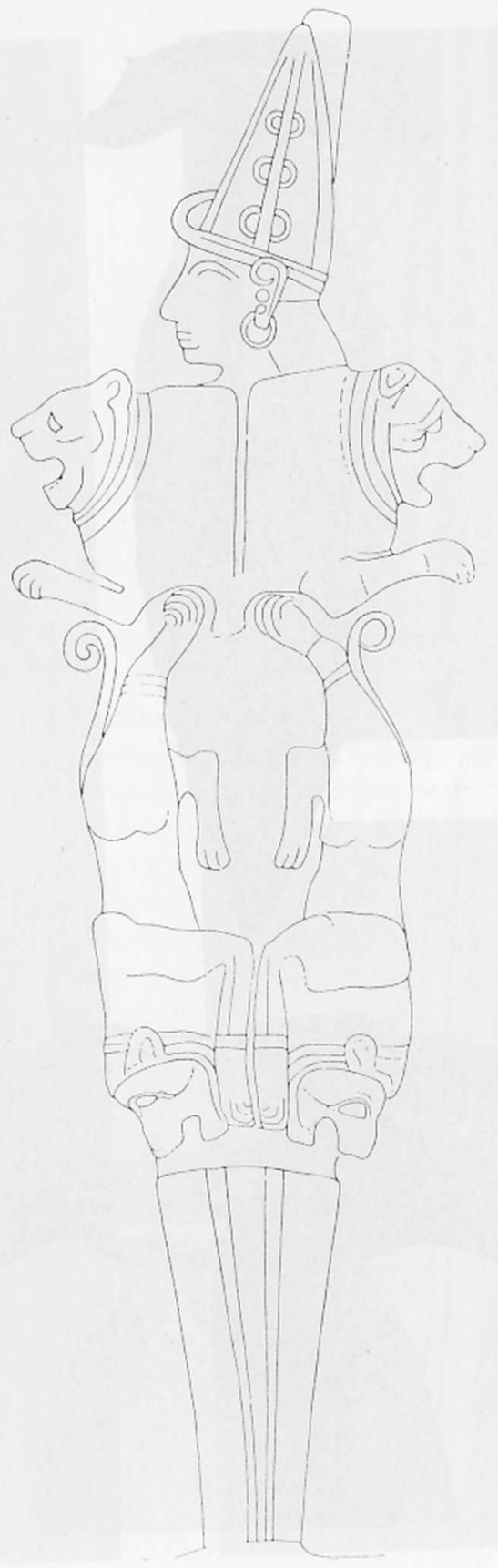

Fig. 1 


$$
\frac{1}{1}
$$




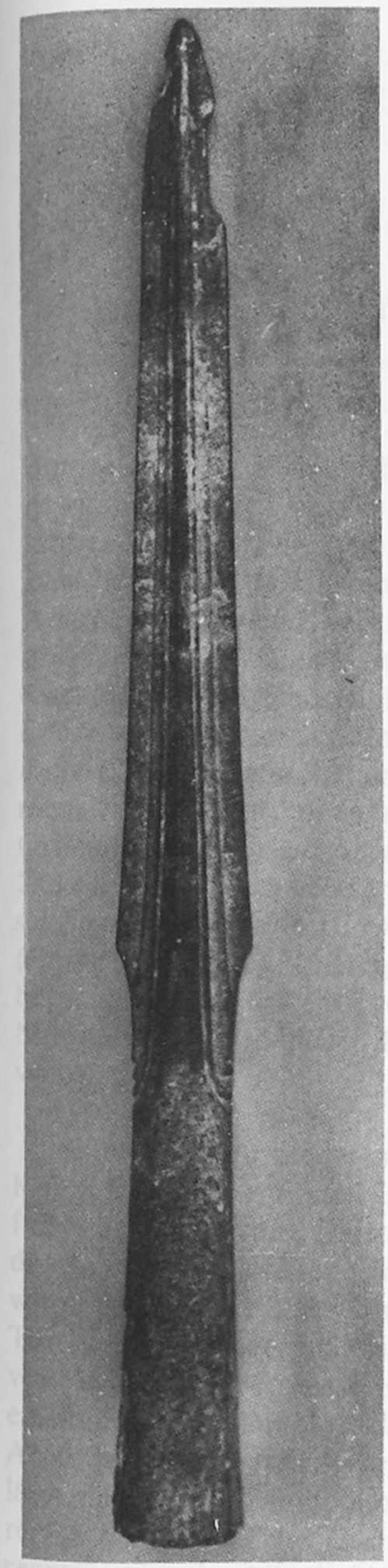

a
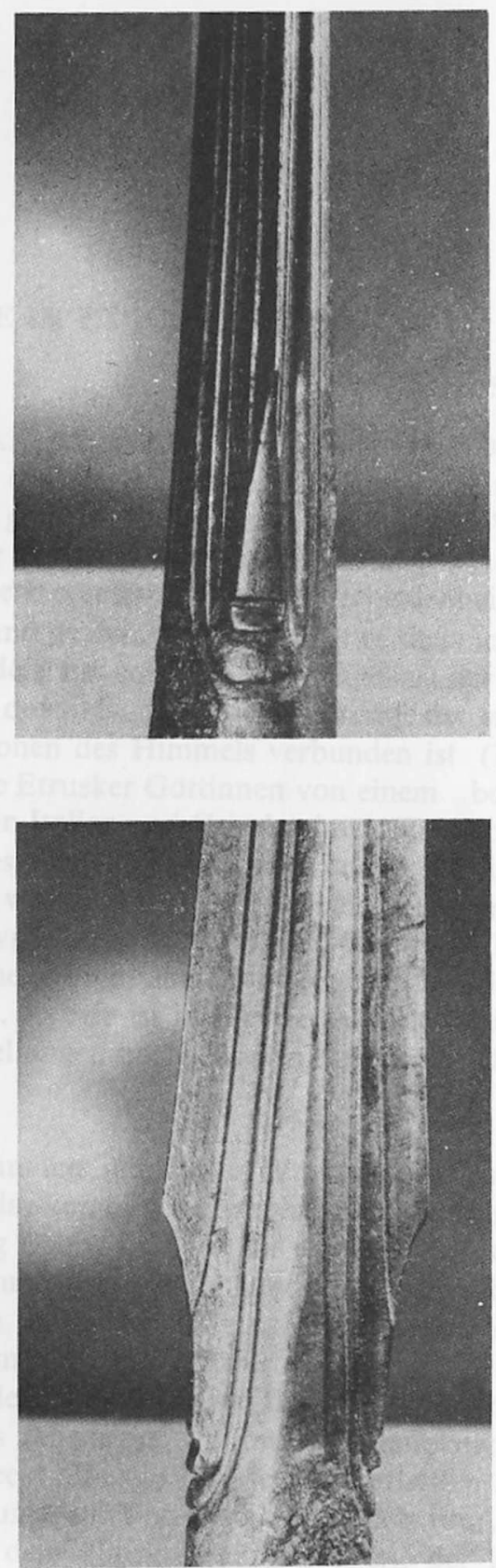

Fig. 3 a-c 